\title{
Mapping Non-Crystalline Nanostructure in Beam Sensitive Systems With Low-dose Scanning Electron Pair Distribution Function Analysis
}

\author{
Joonatan E. M. Laulainen ${ }^{1 *}$, Duncan N. Johnstone ${ }^{1}$, Ivan Bogachev ${ }^{1}$, Sean M. Collins ${ }^{1}$, Louis Longley ${ }^{1}$, \\ Thomas D. Bennett ${ }^{1}$, and Paul A. Midgley ${ }^{1}$. \\ 1. Department of Materials Science and Metallurgy, University of Cambridge, 27 Charles Babbage Road, \\ CB3 0FS, Cambridge, UK \\ * Corresponding author: jeml3@cam.ac.uk
}

Macroscopic properties of modern materials are increasingly determined by the nanoscale arrangement of different structural phases in complex multi-phase nanostructures. To develop nanostructure-property relationships, detailed local characterization methods are required, with most structural insight typically obtained via diffraction techniques [1]. Furthermore, many important non-crystalline or semi-crystalline systems, including metal-organic frameworks (MOFs) and inorganic glasses, are readily damaged when probed by ionizing radiation. Scanning electron diffraction (SED) microscopy has recently emerged as a powerful local diffraction technique capable of providing the necessary characterization and applicable to radiation sensitive materials. In SED, a 2D diffraction pattern is acquired at every pixel as a focused near-parallel electron beam is scanned across the specimen, However, most SED microscopy has been based on analysis of Bragg diffraction and is thus only applicable to crystalline components of nanostructure. Here, we generalize low-dose SED microscopy to address non-crystalline components of nanostructure using pair distribution function (PDF) analysis and demonstrate this approach by application to a beam-sensitive nanocomposite of amorphous ZIF-62 and amorphous $60 \mathrm{NaPO}_{3}-40 \mathrm{AlF}_{3}$.

The PDF quantifies the probability of finding two atoms a distance $r$ apart and can be used to characterize both crystalline and non-crystalline atomic structures [2]. It is typically performed as a bulk method, using X-ray or neutron diffraction, but it is possible to extract PDFs from the azimuthally integrated 1D line profile at each 2D diffraction pattern acquired in a SED microscopy data set, as outlined in [3]. Such analysis is demonstrated in [4,5] and was done using Python3 code written inhouse. The SED microscopy was performed using a JEOL ARM300F operated at $300 \mathrm{kV}$ with a beam diameter of $\sim 5 \mathrm{~nm}$ and a convergence semi-angle of $\sim 0.6 \mathrm{mrad}$. The beam current was $4.6 \mathrm{pA}$, leading to a dose of $\sim 13 \mathrm{e} / \AA^{2}$. The diffraction patterns were acquired using a Medipix 3 direct electron detector with $256 \times 256$ pixels and a maximum scattering vector $q=4 \pi \sin (\theta) / \lambda$ of $17.6 \AA^{-1}$. The limitations of performing PDF analysis using such data was considered in detail and energy-dispersive X-ray spectroscopy (EDX) was performed subsequently to confirm the compositions of the particles.

The 256x256 (in real space) SED data set was cropped and binned 3-fold to make a 56x77 pixel data set. The PDF was then calculated at each binned pixel (consisting of 9 pixels in the original image). To separate the PDF of the MOF and the inorganic glass without prior knowledge, independent component analysis (ICA) was applied. First, a principal component analysis (PCA) singular value decomposition was performed on the PDFs, to calculate orthogonal gaussian signal components. Subsequently, ICA of the 2 largest variance PCA components was calculated to extract independent low-noise PDF signals. Signals corresponding to the MOF and to the inorganic glass were distinguished, as shown in Figure 1c. The ICA loading, a real-space map of similarity between the calculated ICA signal and the PDF at each pixel, was also used as a classifier, to gauge whether the ICA signals correspond to the signals from the regions they represent. The MOF signal was in excellent agreement and the inorganic glass signal agrees 
well except around $3 \AA$. This is likely due to the region of mixed MOF-inorganic glass on the bottom left of the lower particle as seen in the EDX map in Figure 1b and the MOF loading map in Figure 1c. Work to quantify phase fractions in the mixed region using relative pdf intensities is underway.

A mixture of two non-crystalline nanoparticles was mapped at low doses using PDF analysis and ICA in STEM. Distinct signals in the ICA decomposition of the PDF show that it is possible to use this method to map beam-sensitive amorphous nanomaterials with a few nanometers spatial resolution without prior knowledge of the structural domains present [6].

\section{References:}

[1] SJL Billinge and I Levin, Science, 316 (2007) p. 561.

[2] DJH Cockayne, Annual Review of Materials Research, 37 (2007) p. 159-187.

[3] DJH Cockayne and DR McKenzie, Acta Crystallographica Section A, 44.6 (1988) p. 870-878

[4] P Lu and BD Gauntt, Microscopy and Microanalysis, 19.2 (2013) p. 300-309.

[5] X Mu et al, Ultramicroscopy, 168 (2016) p. 1-6.

[6] The authors acknowledge funding from the EPSRC (Grant no. EP/R008779/1) and through doctoral training award EP/R513180/1. We thank Diamond Light Source Ltd. for access and support in the use of the electron Physical Science Imaging Centre (EM16983, EM19130, EM20195, MG21979).
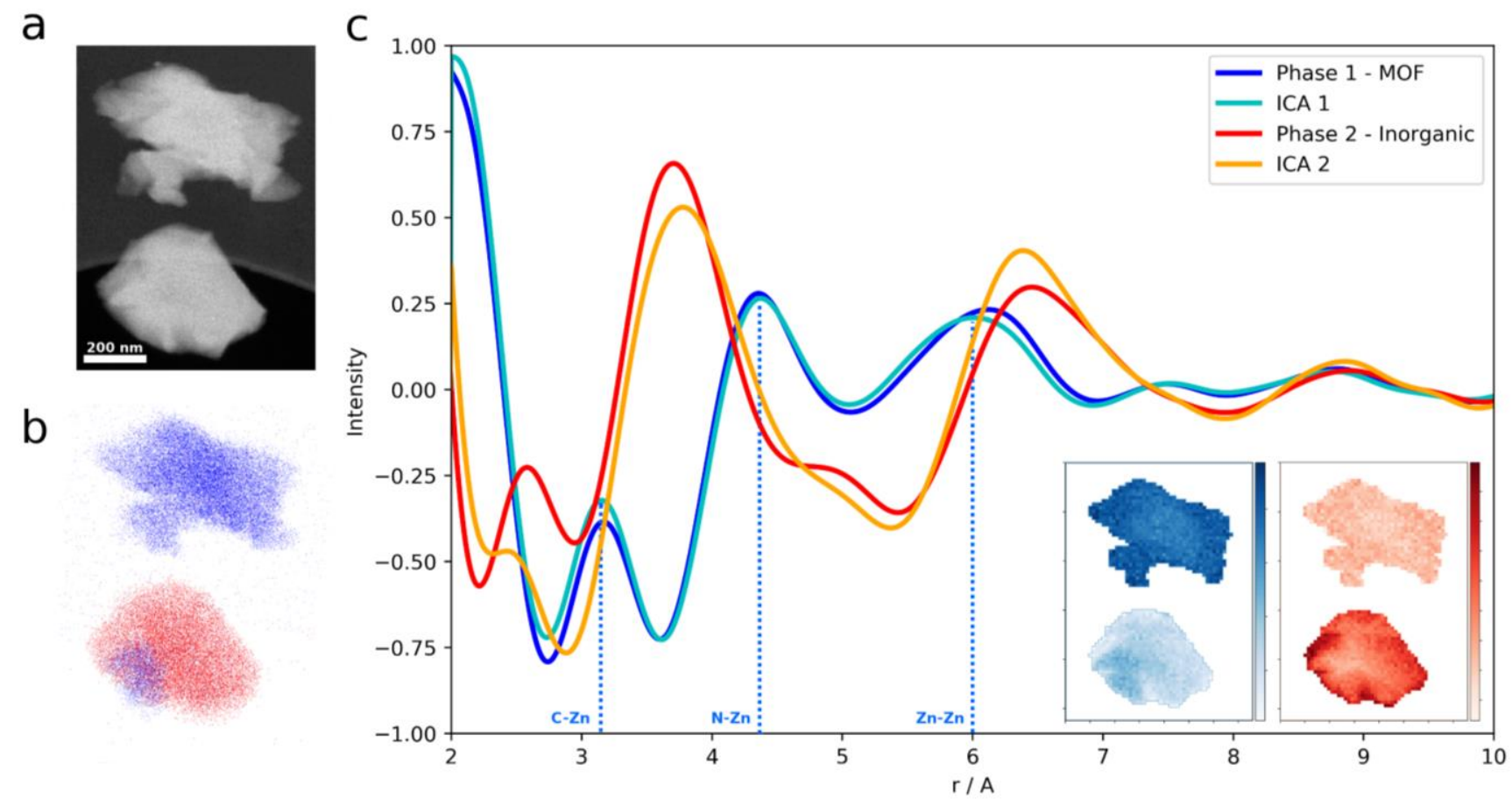

Figure 1. Mapping of an amorphous ZIF-62 glass and an inorganic $60 \mathrm{NaPO}_{3}-40 \mathrm{AlF}_{3}$ glass nanoparticle mixture using PDF-STEM. (a) Virtual ADF image of the two particles. Inner and outer radii were $\mathrm{q}=$ $10.0 \AA^{-1}$ and $q=17.6 \AA^{-1}$. (b) EDX map of Zn (blue) and Al (red) taken after the STEM data. Note a small inclusion of ZIF-62 in the lower particle. (c) ICA components of the two observed phases (ICA1 and ICA2) and summed signals from the areas with the highest $40 \%$ intensity in the loading map (bottom right) for each ICA component. These were classified as Phase 1 (MOF) and Phase 2 (inorganic glass), for which an average PDF was also obtained (red and dark blue). The peaks in the MOF PDF are labelled with the corresponding atomic distance in the structure. 